\title{
Determining Trust Based Examination on Social Networks for Hotel Recommendation
}

\author{
Shri Vindhya, V.Dharnesh
}

\begin{abstract}
The objective of this research work is to enhance the performance of a novel recommendation site for tavern by mining the data about all kinds of hotel in websites. When we are about to view the rating of a hotel given by other people, we can't rely accurately on it as there are many fake reviews. So we can overcome the problem by viewing only the ratings given by our friends and friends of friends in our trusted network. TrustSVD algorithm considered for ensuring this. By considering both the implicit and explicit opinions of ratings the predictions are made. The proposed technique is used to merge with social trust information thus from that we can get the trusted network. Thus, by using the trusted network reviews we can avoid the fake reviews. In case of cold start and data sparsity problem the friends of friends list is considered.
\end{abstract}

Keywords: TrustSVD, Ratings, Social Trust

\section{INTRODUCTION}

When we are about to view a rating of a hotel given by other people, we can't rely accurately on it. Thus, a TrustSVD++ algorithm (explicit and implicit effect) and the trust on rating is used. Those trust network is a small network which incorporates trust based rating system. Trust Network is used because memory based systems are not applicable for large data sets. Thus proposed model a novel recommendation site along with a social networking site is used. Various sites like Facebook, Instagram, Google + are being widely used by many people. OSN's are a great platform to disseminate information, sharing information, poll answering etc First contribution is to conduct empirical analysis on trust and ratings to find relationship between them. The users are strongly correlated or weakly correlated to each other. The social relationship of user is different based on that the relation varies. Thus, the social relationship makes us to consider both the explicit influence and implicit influence. The implicit influence gathered from trustier and trustee is used to generate analysis vector. The explicit influence is used to factorize the trust matrix into trust specific vectors and trustee specific vectors. Thus, the ratings are bridged into a unified model. The third contribution is to do the experiments to find the effectiveness of the proposed system.

Revised Manuscript Received on July 13, 2019.

Shri Vindhya, Associate Professor, Faculty of Engineering, Saveetha School of Engineering, Saveetha Institute of Medical and Technical Sciences, Thandalam, Chennai, Tamilnadu, India-602105

V.Dharnesh, UG Scholar, Saveetha School of Engineering, Saveetha Institute of Medical and Technical Sciences, Thandalam, Chennai, Tamilnadu, India- 602105

This can be Done by using different types of testing view. This process is done for all user and cold start user. This testing view is used to predicate the accuracy. First one is all view in which all the User who are involved in recommender system is included. Second is cold start view in which the users who have rated the items less than five are involved. There are many issues as below, Cold start is a major issue in a recommender system where there are only few numbers of users who rate. Thus, cold start is a major issue which has to be sorted out in a recommender system. Scalability is an important point to be noticed. Scalability is the practicality with which the process is implemented. Hence, a recommender system must be scalable enough for a creating a recommendation model. Recommender system must be applicable for a long tail. The data must be completely updated every time on a database. Hence, it must be done in a unique process. Hence, it is possible to trust a rating given by a user. Novelty and diversity of recommendation: There must be diversity in the recommendation offered by a recommender system so that the user can choose his requirements specifically. Sparse, Missing, Erroneous and Malicious data in which the proposed system must be reluctant to malicious data and missing data such that the users are provided with a rating in a more specific and reliable content is provided. Conflict resolution while using ensemble hybrid approaches in which there may occur conflicts when recommending a certain item. Hence, the recommender system must be able to address the situation. Hence, the conflicts must be avoided. Ranking of the recommendations in which the ranking of the recommendations must be done in such a way that all the user requirements are satisfied while designing a recommender system. Impact of context-awareness in which the recommendation suggested must be a context aware result. Otherwise, the recommender system may lose its properties. Impact of mobility and pervasiveness involves impact of mobile computing must not be seen in recommender system. It must be compatible with all kinds of hardware and software.

\section{DEFINITION}

Trusted rating plays a major role in e-commerce activities such as navigation of product offerings, personalization, improvising Customer reliability and in marketing. The trust based system is implemented by using the collaborative algorithm. By using $\mathrm{CF}$ we can predict the taste of user in future based on his taste in the past. This can be done by finding the similar taste user and suggesting his preferences. $\mathrm{CF}$ has also been used in the field such as image processing and bio-informatics. 


\section{Determining Trust Based Examination on Social networks for Hotel Recommendation}

However, Collaborative Filtering suffers from two issues data sparsity and cold start. The first issue is that the ratings given by the existing user is very less which means they rate only a little portion. The second issue is that the new user will give only few ratings. This issues leads to data inadequate. Both the issue will reduce the accuracy of the recommender system. The ratings for the not rated items are predicted in the proposed system. To overcome these two issues we consider the social trust information. Thus, by using the social information the similar taste user can be identified. The top recommendation will be high ratings given by that similar taste user. If a user search for the particular hotel then the ratings given by his friends are visible. If the user search for the recommendation of the hotel based on the taste the hotel get recommendation. The recommendation details are gathered from the trusted network user, if friends list is not sufficient to recommend a hotel then the friends of friends list are considered from the trusted network.

\section{BACKGROUND}

\section{Rating Prediction}

G. Guo et al (2012) tells that the user rating profile can be merged with the user of the trusted network for the betterment and also tells that by doing so the cold start problem and data sparsity problem is overcome. However, memory based methods has a disadvantage in adapting to the large datasets and the time consumed to search the candidate in the large data set is more. The model based method can handle the large data set and item prediction is accreted. The model based approach has higher accuracy rate and higher resistance to data sparsity problem compared with that of memory based approach tells Y. Koren et al (2009). Instead of predictions of items property of trust propagation are enabled.

\section{Item Recommendation}

A short review is given on the recommendation of item based on the trust based model. Q. Yuan et al (2011) combine friendship and membership in a unified matrix by a factorization method. These two types of relationships are different. In this article we consider either one of the social relationship that is trust and trust alike. But we show the application of both the relationship on the model S Rendle \& C Freudenthaler (2009) gives state of art model called Bayesian personalized ranking (BPR), for recommendation of items based on feedback that are implicit. The active users rated item is generally considered as unrated item. The negative samples are considered as unawareness of item rather than dislikes. To overcome assumptions W. Yao et al (2014).He propose a Social Bayesian personalized ranking (SBPR) method which tells that the item consumed by the user is considered as consumed by his friends which on later considered as consumed by other users. The item recommendation wants both the positive sample and negative sample whereas the rating prediction needs only the positive samples. The recommendation of items is evaluated based on the list of ranking of performance but the rating prediction is evaluated based on the difference between the ground truth and prediction, although the item

recommendation is not so accurate it still predicts the ratings that are not given by the user. Our work is focused only on the rating prediction rather than item prediction because the former is very effective.

\section{Data Sets}

For the process of investigation we conduct the analysis on four real world data sets. These data sets are FilmTrust, Epinions, Flixster and Ciao. First thing is that the available trust information is really very less. Second is that the user is strongly bonded with the outgoing user who are trusted. But they are weakly bonded with that of the trust alike user. The third observation is that for the incoming trust users can also get the similar conclusion. It won't be that much effective if it considers only the trust alike relationship. The data sets such as FilmTrust, Epinions, Flixster and Ciao are used in analysis which contains both social relationship and item rating. The items in Epinions, Ciao are the huge variety of electronic items, sports related items. Item in FilmTrust and Flixster contains only films. The ratings in Epinions and Ciao are in the form of integers that are given from one to five. In the other data sets the user can share their ratings of the items with each other. Then based on the similar taste user is identified and used in the process of recommendation. The relationship in Epinions and Ciao are termed as trust relationships which construct social network. The relationship in Flixster and FilmTrust are termed as trust alike relationship. Further, the users in Epinions and Ciao specify the users as the trusted one based on their qualities and reviews made by them. Flixster uses the process of friend network, where relation of user is symmetric and rating is done for movies only. FilmTrust uses the concept of trust and it uses binary values thus the trust alike is considered rather than trust.

\section{PROPOSED WORK}

The recommendation model that is based on trust and which consider both user and item rating is called trustSVD. This approach is on the top of the art state model SVD++ in which the explicit and implicit influence user ratings both are used on items that are involved to produce predictions. The ratings of user are given mostly in the pictorial form that is graphical form. In the friend of friend recommendation model used overcome the data sparsity and then cold start issues. Thus, the mining process produces result to user graphical representations, in the form of overall rating graphical representation and show the relationship between the user and rated user and the individual rating of the particular user graphical representation. Online social network can be used for chatting, sharing post and multimedia communication. In our proposed system we mainly use a collaborating filter which means it is used to integrate the information of multiple users and their taste are calculated. This filtering process is used to filter the users from the large number of users. This user will get recommendation based on the filtering process. The main aim of our paper is to consider the implicit and explicit ratings. 
The implicit ratings are directly given by the user whereas the explicit ratings are given based on the interest of the user. The existing method suffers from two problems. These cold start and data sparsity problem is overcome by considering the friends of friends list. In the recommendation site the ratings are done based on the expectation of that person and then there are also many fake rating. Thus, the trusted rating can be obtained using trust based matrix factorization for recommendation. It makes use of SVD++ recommendation algorithm. The user's social network site will be merged with the novel recommendation site. The rating done by the users are stored in the database. Both the explicit and implicit influence of rating is considered. The user can make decision by using the rating done by his friends in social media. These rating are considered as trusted rating. The user can view the rating from his friend list in social network. Then the mining is done to filter the item and then display it to user. From that displayed item he can select the particular item and also he can see the different perspective rating of that particular item. Looking only into friend list sometimes may not give any matching giving rise to data scarcity and cold start problem. To overcome this we can see into friend of friend list. After mining the information is represented in the graphical form. It shows the graphical representation between user, rated user and individual rating. Based on this graphical rating the user chooses the items. The main domain of the method is data mining.

\section{PERFORMANCE ANALYSIS}

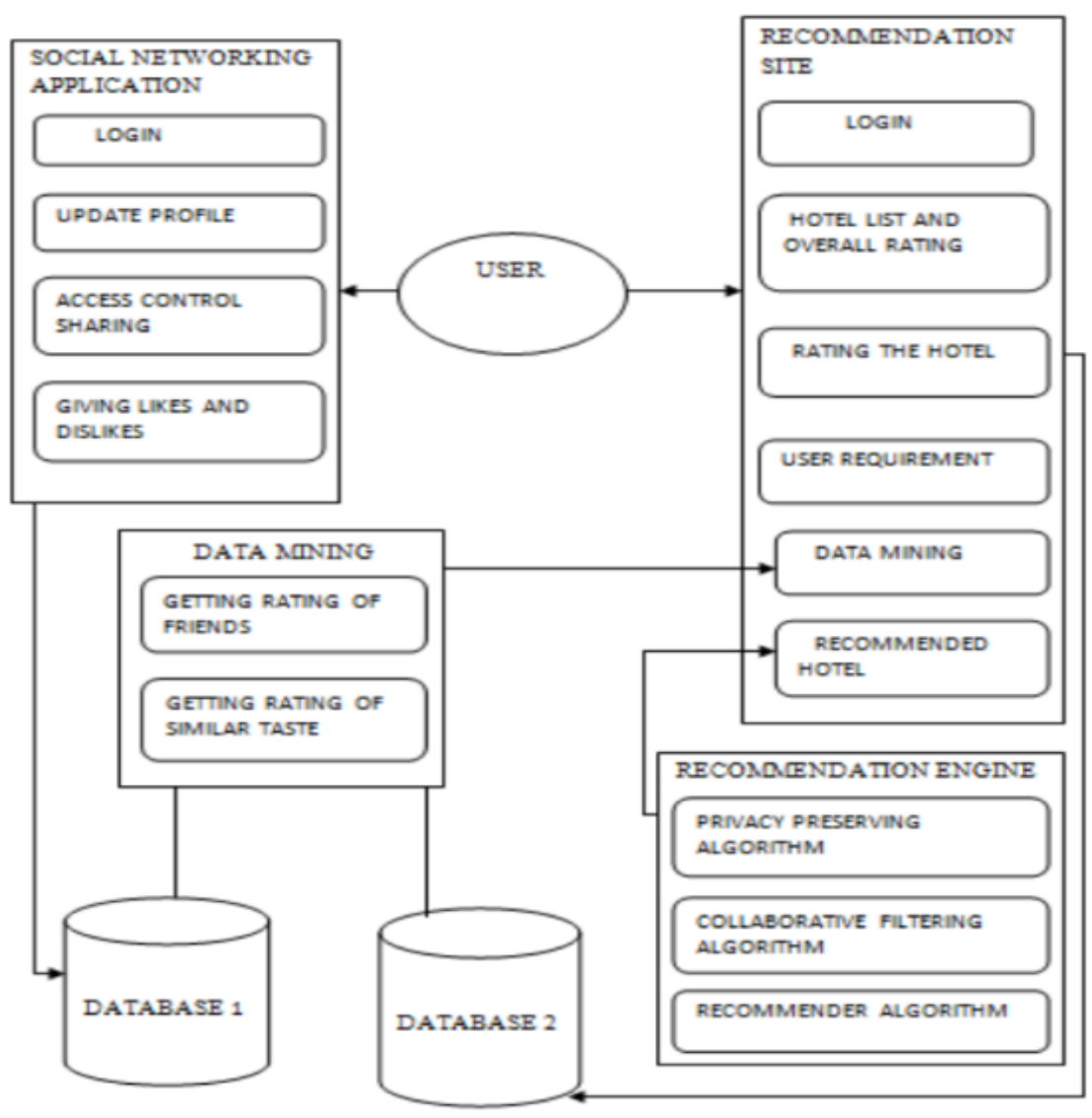

Fig. 5.1 Architectural Design

\section{Techniques Used}

In this proposed model a novel recommendation site along with a social networking site is used. For this purpose three primary algorithms are used, they are Collaborative filtering algorithm, Recommender systems algorithms, Distributed and Privacy Preserving. The use of collaborative algorithm is Automatic prediction is made about the interest of the user. This works by gathering the taste and preference of the many users. The users are categorized based on the ratings done by them. The rating may be done to any items like book, dress, film and more. Based on the rating the similar taste users are identified thus the user will recommendation the item to which the similar taste user has rated highly. matrix is developed based on the relation between by using Collaborative filtering. In the miner.

\section{Architectural Design}




\section{Determining Trust Based Examination on Social networks for Hotel Recommendation}

In Fig. 5.1 architecture design there are four main modules and they are trusted network, recommendation site, recommendation engine and data mining. The trusted network is formed by using the social networking application and the recommendation site is with normal performance. During recommendation the recommendation engine makes use of the filtering process to filter the hotels. The process has two databases, one is to hold the value of the trusted network and the other is to hold the rating values. The user matrix and user item matrix are developed based on the information that is available on the database.

\section{Work Flow}

The proposed recommendation site has the pre-processing activities and then the overall rating of the hotels. The overall rating of the hotel is filled by using cumulative values. The recommended system is merged with the social media application. Using the social media the trusted network is formed. Then rating has done by the trusted friends and friends of friends play the vital role. In the recommendation system two matrices are used. The first one is the user-user matrix which get filled using the trusted network, if user is in the trusted network then filled by one else filled by zero. The second one is user item matrix. If the user had given rating to that item then it gets filled by that value otherwise using collaborative filtering algorithm the matrix is filled. The user can either search the rating for the particular hotel or he can get the suggestions for the hotels. The higher priority of the similar taste user is suggested. Ten the recommendation algorithm is used to suggest the highly rated hotel to the user.

\section{TrustSVD Model}

In the social networking application trusted network is formed by adding friends whom we trust. Also, the trusted network is not symmetric in nature if a user is present in the trusted network of other user then it does not mean that the other user also in the trusted network of that user. Thus, the user cannot be in the trusted network of the other user unless he adds that user. The user can rate the items from one to five integers. The item can be anything like film, gadgets, and hotels. Based on the rating the similar taste user is identified. Based on the rating of the user the item gets recommended. If the user not rated then the rating is predicted based on the preference by using user matrix and user item matrix. The rating for item $\mathrm{j}$ on user $\mathrm{u}$ is predicted by, Implicit influence of the trusted user is given by the equation, Implicit influence of the trusting user is predicted by, The two implicit influence are linearly combined using the following equation, The over fitting problem is the major issue which is avoided by using the weighted lamda regularization method. Then the learning algorithm is framed for the efficient work. This algorithm takes the input as user matrix, user item matrix, regularization parameters. The output of the parameter is the rating prediction. Initially the parameters are initialized with random and small values. Finally, the learned vector is returned as the output. The trustSVD model uses both the explicit and implicit influence of the user rating. The learning in the trustSVD model is given by the algorithm.

\section{CONCLUSION}

The proposed novel recommendation model has concluded that there should be trusted and rating. By the analyze of four real data set it is concluded that the trust and ratings are complement to each other In the trust model we consider both the explicit and implicit influences. And also the influence of both the tuster and trustee is considered. The weighted-lamda-regularization method is used in the generation of latent vector of user and item. It is known that the social trust information of the user can improve the accuracy of the recommendation. The recommender system is based on item recommendation and rating prediction. The algorithm can be designed only for anyone and we focus on rating prediction. The proposed model is used to overcome cold start problem and data sparsity problem.

\section{REFERENCES}

1. B. Yang, Y. Lei, D. Liu, and J. Liu 2013.Social collaborative filtering by trust, In Proc. 23rd Int. Joint Conf. Artif. Intell., pp. 2747-2753.

2. G. Guo, J. Zhang, and D. Thalmann 2012. A simple but effective method to incorporate trusted neighbors in recommender systems, In Proc. 20th Int. Conf. User Model., Adaptation Personalization, pp. 114-125.

3. J. Tang, X. Hu, H. Gao, and H. Liu 2013. Exploiting local and global social context for recommendation, In Proc. 23rd Int. Joint Conf. Artif. Intell., pp. 2712-2718.

4. M. Jamali and M. Ester 2010. A matrix factorization technique with trust propagation for recommendation in social networks," in Proc. 4th ACM Conf. Recommender Syst., pp. 135-142.

5. Q. Yuan, L. Chen, and S. Zhao 2011. Factorization vs. regularization: Fusing heterogeneous social relationships in Top-n recommendation, In Proc. 5th ACM Conf. Recommender Syst., pp. 245-252.

6. S. Rendle , C. Freudenthaler, Z. Gantner, and L. Schmidt-Thieme 2009. BPR: Bayesian personalized ranking from implicit feedback, In Proc. 25th Conf., Uncertainty Artif. Intell., pp. 452-461.

7. W. Yao, J. He, G. Huang, and Y. Zhang 2014. Modeling dual role preferences for Trust-aware recommendation, In Proc of 37th Int. ACM SIGIR Conf . Res. Develop. Inform. Retrieval, pp. 975-978.

8. Y. Koren, R. Bell, and C. Volinsky 2009. Matrix factorization techniques for recommender systems, Computer, vol. 42 , no. 8 , pp. 30 37, Aug. 